\title{
Ambulatory Monitoring of Performance and Health in the Military using Field Labs
}

Operations in Iraq, Afghanistan and Bosnia reveal that soldiers are exposed to extreme, complex and sustained operations, crossing the limits of human capabilities. During these operations the operational readiness or status of the soldier is estimated by the commander based on subjective observations of the physical and mental capabilities of the soldier. This commander evaluation can be biased by personal experiences and commanders own physical and mental status.

Wrong judgement can have negative consequences for the soldier, which might lead to dangerous and harmful situations. To predict the operational readiness and to guard the safety and health of soldiers, it is important to objectively recognize symptoms of fatigue and exhaustion. Up till now no methods were available for the Dutch Army

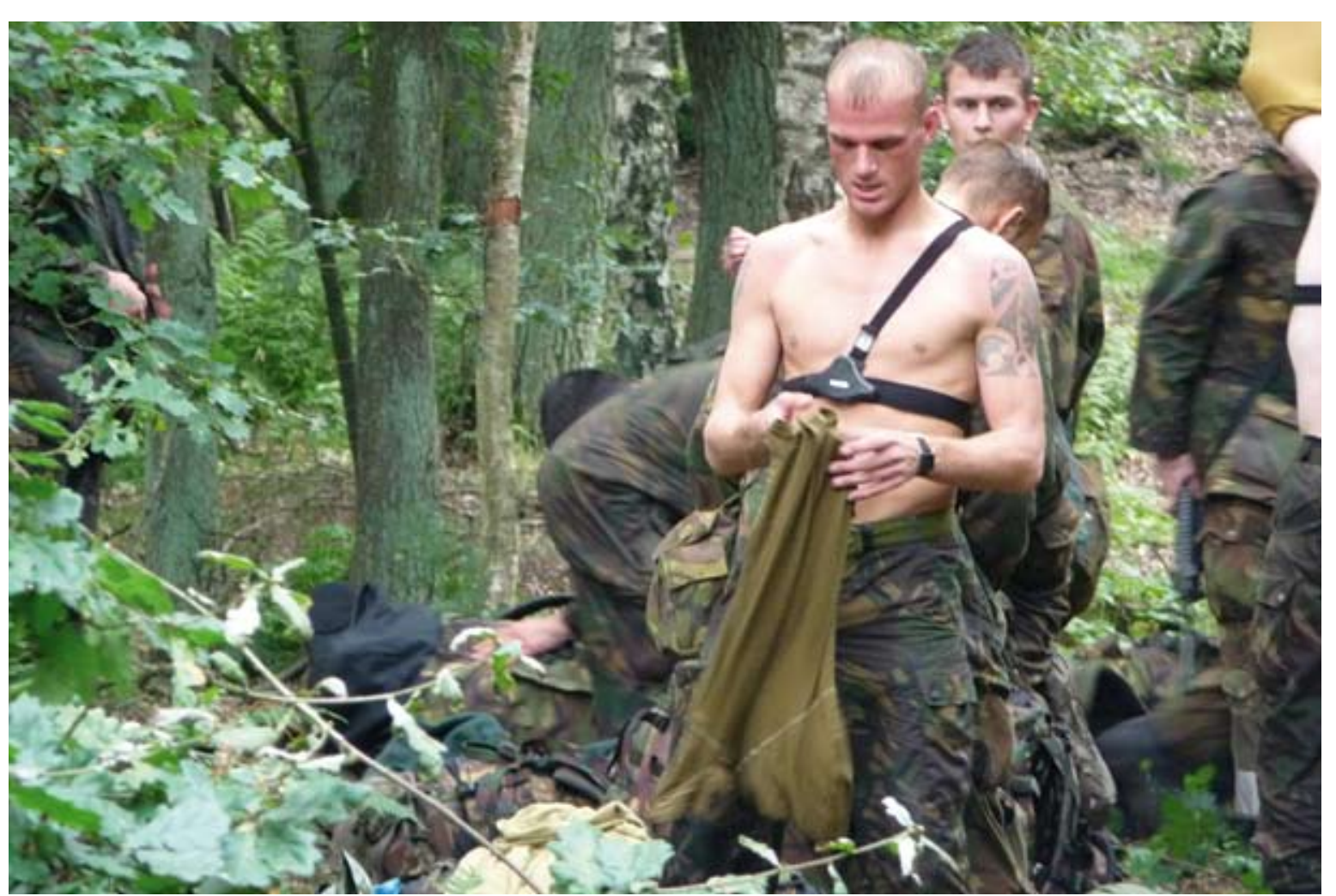
soldiers during military operations.

\section{PURPOSE}

Monitoring the physical and cognitive performance of Monitoring the physical and cognitive performance of
soldiers during an Air Mobile Brigade training course.

\section{to monitor physiological and cognitive status of \\ METHODS}

56th Annual Meeting of the American College of Sports Medicine (ACSM), May 27
A field lab was developed to assess soldiers physiological and cognitive functioning during a 7-week training course. During 3 weeks physiological parameters like heart rate, skin- and core temperature, respiration rate and activity were measured 24 hours a day during 3 days for 3 weeks. Cognitive performance (logical reasoning, working memory and vigilance) was measured 5 times a week using the N- Back task, Tower of Hanoi and the VigTrack task. Short questionnaires assessed the amount and and subjective alertness.

RESULTS

The physiological measurements indicate Heart Rate Reserve percentages up to $80 \%$. Core temperatures sometimes reached values up to 39 degrees (averaged per hour). Cognitive performance was seriously affected in the second week. Reaction time decreased (N-Back, VigTrack) while initiation time (Tower of Hanoi) and errors increased.

\section{CONCLUSIONS}

Soldiers were not able to maintain optimal physical and cognitive functioning during the training course. Intermittent low impact weeks appeared to be sufficient to restore cognitive functioning. quality of sleep, level of exertion, need for recovery
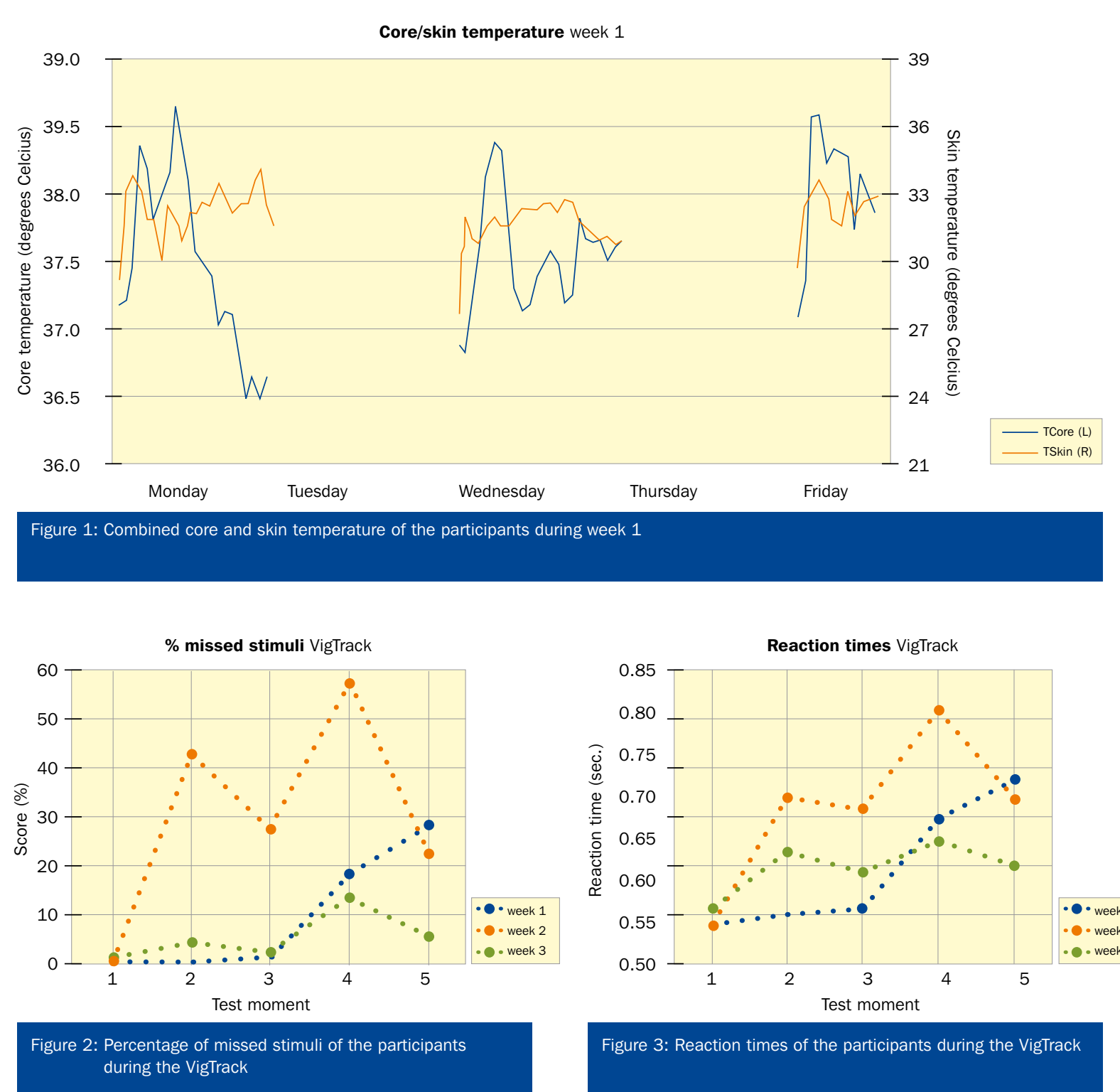